\title{
A new method of metabarcoding Microsporidia and their hosts reveals high levels of microsporidian infections in mosquitoes (Culicidae)
}

\author{
Artur Trzebny ${ }^{1}$, Anna Slodkowicz-Kowalska ${ }^{2}$, Becnel James ${ }^{3}$, Sanscrainte Neil ${ }^{3}$, and \\ Miroslawa Dabert ${ }^{1}$ \\ ${ }^{1}$ Adam Mickiewicz University \\ ${ }^{2}$ Poznan University of Medical Sciences Faculty of Medicine I \\ ${ }^{3}$ USDA-ARS Center for Medical Agricultural and Veterinary Entomology
}

April 28, 2020

\begin{abstract}
Microsporidia are obligate intracellular eukaryotic parasites that infect nearly all animal groups, including humans. The most common molecular methods for Microsporidia detection rely on species-targeting qPCR or end-point PCR using group-specific primers. However, these methods could be not specific enough or fail in case of mixed infections. We developed a method for parallel detection of both microsporidian infection and the host species. We designed new primer sets: one specific for the classical Microsporidia (targeting hypervariable V5 region of ssu rDNA), and a second one targeting a shortened fragment of the COI gene (standard metazoan DNA-barcode); both markers are well suited for a NGS approach. The analysis of ssu rDNA dataset representing 607 microsporidian species (120 genera) indicated that the V5 region enables identification of $>98 \%$ species in the dataset (596/607). To test the method, we used microsporidians that infect mosquitoes in natural populations. Using mini-COI data, all field-collected mosquitoes were unambiguously assigned to seven species; among them almost $60 \%$ of specimens $(127 / 212)$ were positive for at least 11 different microsporidian species, including a new microsporidian ssu rDNA sequence (Microsporidium sp. PL01). Phylogenetic analysis of Microsporidium sp. PL01 ssu rDNA showed that this species belongs to one of the two main clades in the Terresporidia. In addition, the level of microsporidian mixed infections was relatively high (9.4\%). The numbers of sequence reads for the OTUs suggest that the occurrence of Nosema spp. in co-infections could benefit them; however, this observation should be re-tested using more intensive host sampling. The proposed method for detection of Microsporidia can be applied to all types of DNA extracts, including medical and environmental samples.
\end{abstract}

\section{Hosted file}

Trzebny_resubmit2.pdf available at https://authorea.com/users/316305/articles/446468-a-new-methodof-metabarcoding-microsporidia-and-their-hosts-reveals-high-levels-of-microsporidian-infectionsin-mosquitoes-culicidae 


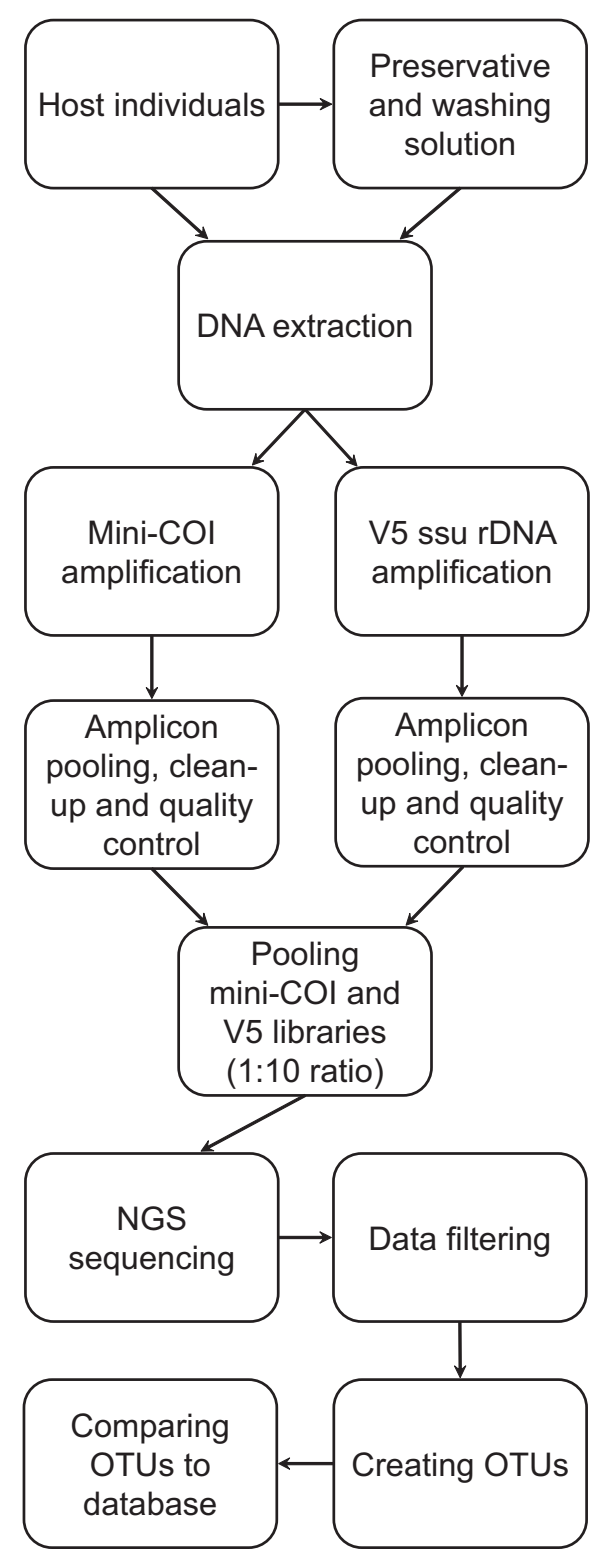




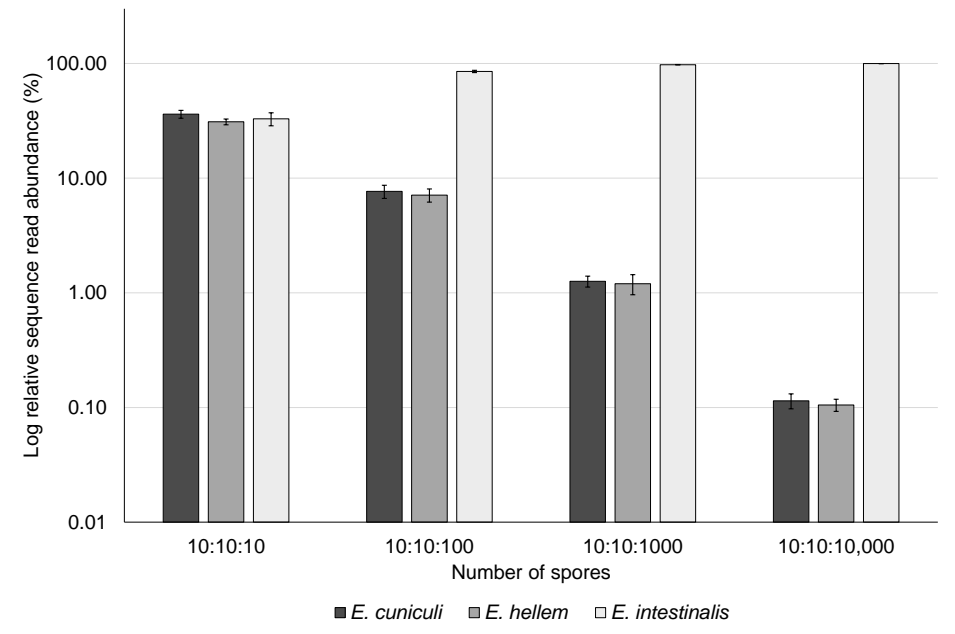




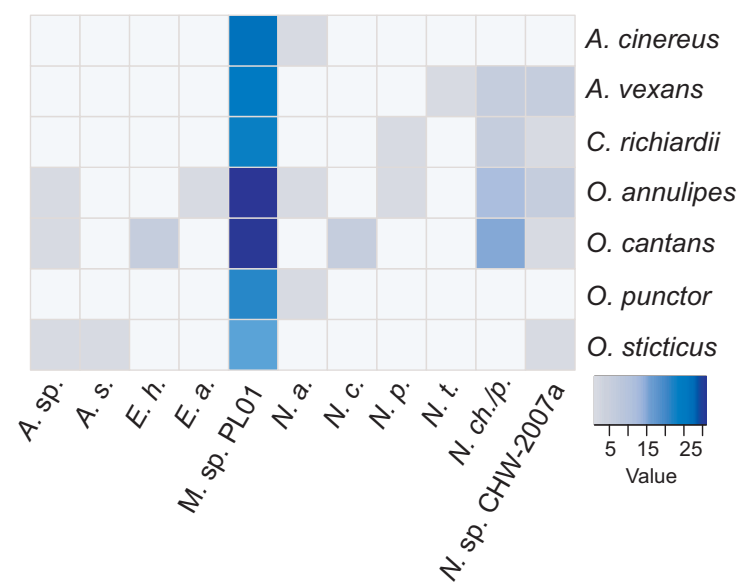




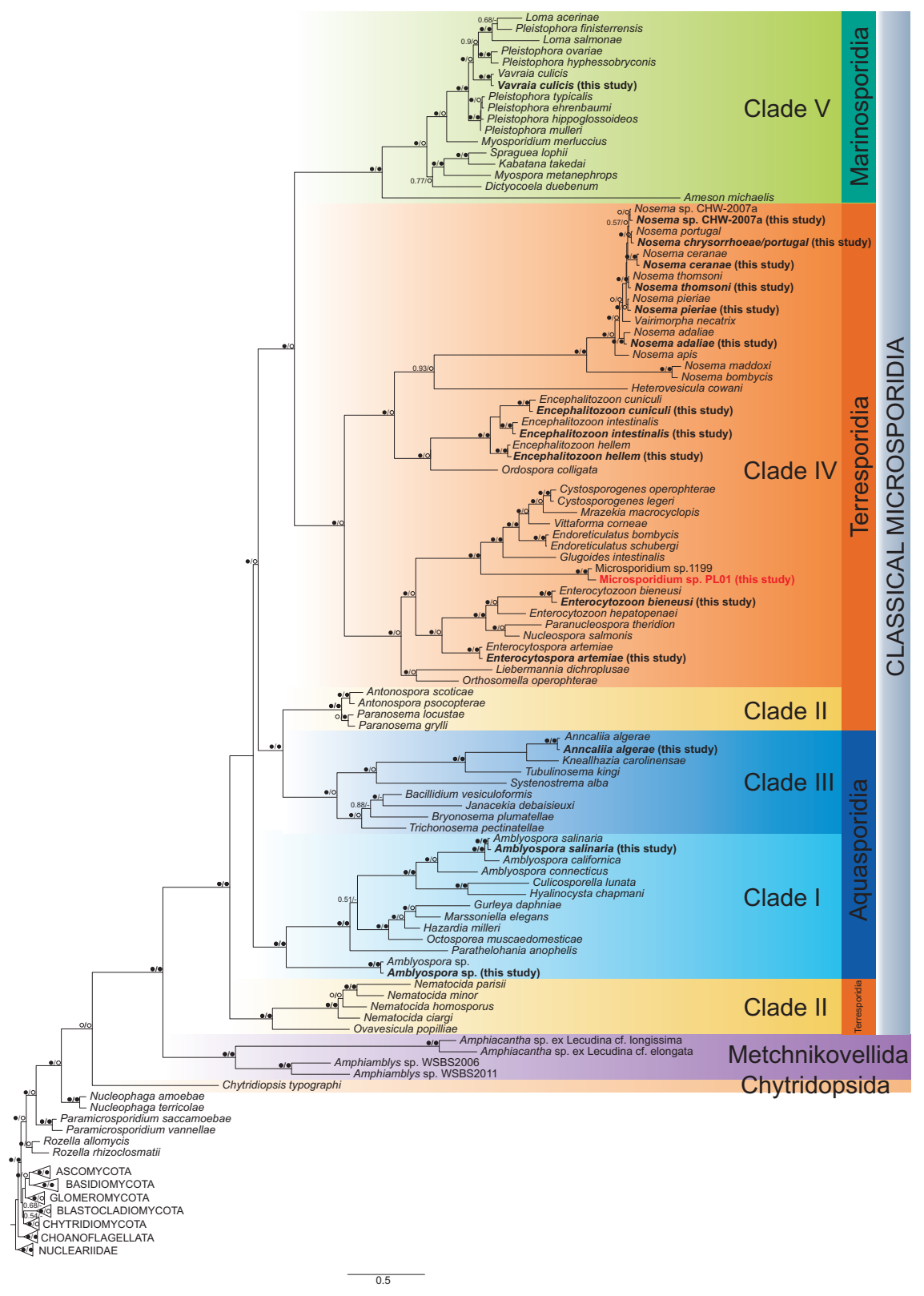




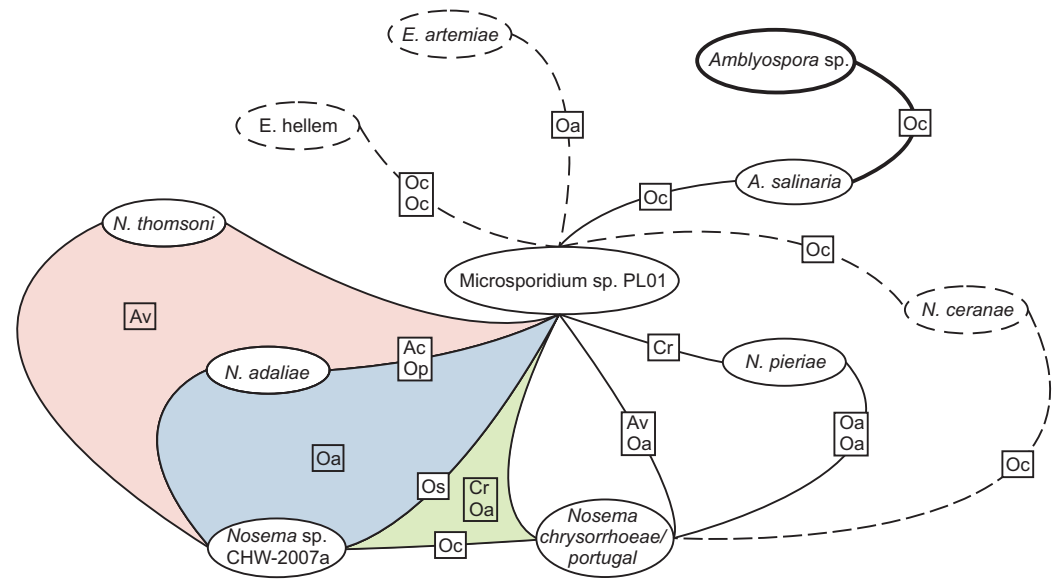




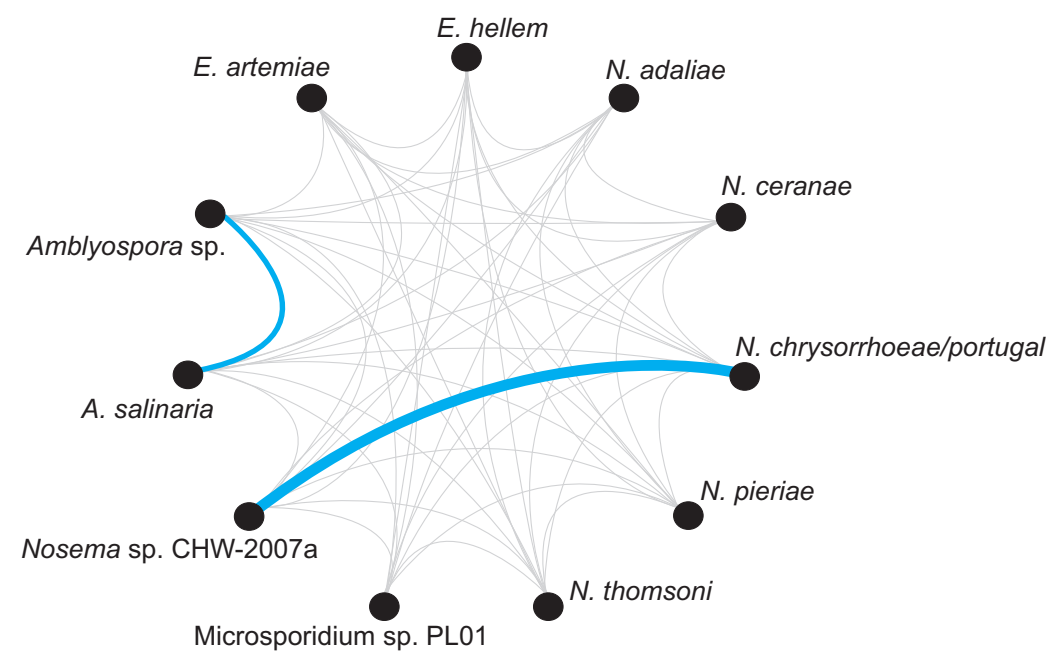



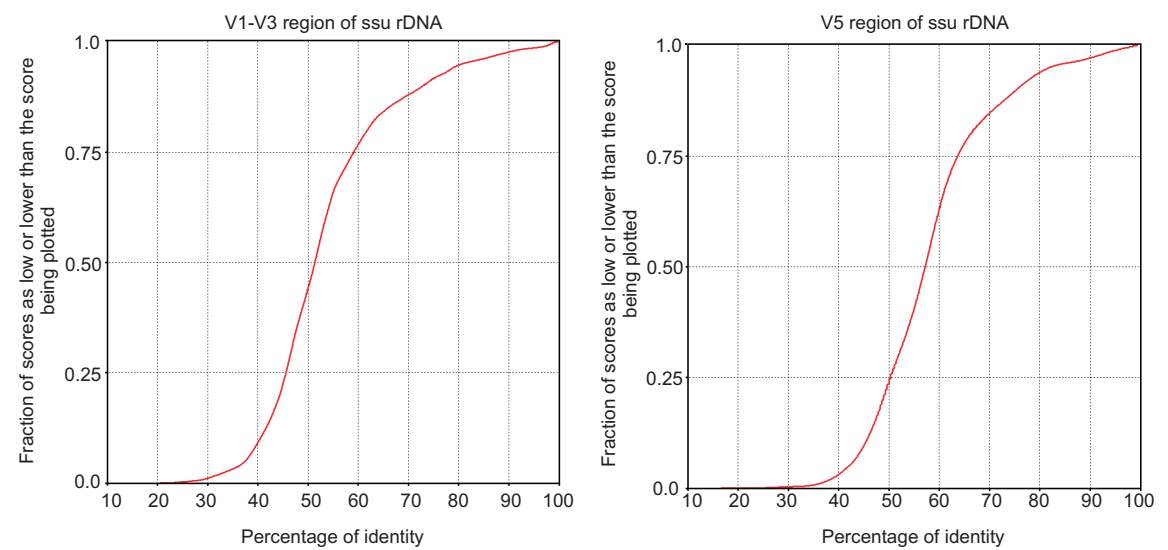

\begin{tabular}{|c|c|c|c|c|c|c|c|c|c|c|}
\hline \multirow{2}{*}{ Microsporidia } & \multirow{2}{*}{$\begin{array}{c}\text { A. cinereus } \\
92.3 \% \\
(n=13) \\
\end{array}$} & \multirow{2}{*}{$\begin{array}{c}\text { A. vexans } \\
63.2 \% \\
(\mathrm{n}=19) \\
\end{array}$} & \multirow{2}{*}{$\begin{array}{c}\text { C. richiardii } \\
56.3 \% \\
(\mathrm{n}=16)\end{array}$} & \multirow{2}{*}{$\begin{array}{c}\text { O. annulipes } \\
54.0 \% \\
(n=63) \\
\end{array}$} & \multirow{2}{*}{$\begin{array}{c}\text { O. cantans } \\
57.1 \% \\
(\mathrm{n}=77)\end{array}$} & \multirow{2}{*}{\begin{tabular}{|c|} 
O. punctor \\
$72.7 \%$ \\
$(\mathrm{n}=11)$
\end{tabular}} & \multirow{2}{*}{$\begin{array}{c}\text { O. sticticus } \\
61.5 \% \\
(n=13)\end{array}$} & \multirow{2}{*}{$\begin{array}{c}\text { All } \\
59.9 \% \\
(n=212)\end{array}$} & \multicolumn{2}{|c|}{$\begin{array}{l}\text { Prevalence (\%) } \\
\text { of microsporidian } \\
\text { species }\end{array}$} \\
\hline & & & & & & & & & in all & in $M-P$ \\
\hline Amblyospora salinaria & & & & & & & 1 & 1 & 0.47 & 0.67 \\
\hline Amblyospora sp. & & & & 1 & 1 & & 1 & 3 & 1.42 & 2.00 \\
\hline Encephalitozoon hellem & & & & & 2 & & & 2 & 0.94 & 1.33 \\
\hline Enterocytospora artemiae & & & & 1 & & & & 1 & 0.47 & 0.67 \\
\hline Microsporidium sp. PL01 & 12 & 10 & 9 & 31 & 27 & 8 & 7 & 104 & 49.1 & 69.33 \\
\hline $\begin{array}{l}\text { Nosema adaliae } \\
\end{array}$ & 1 & & & 1 & & 1 & & 3 & 1.42 & 2.00 \\
\hline N. ceranae & & & & & 2 & & & 2 & 0.94 & 1.33 \\
\hline N. chrysorrhoeae/portugal & & 2 & 1 & 4 & 16 & & & 23 & 10.8 & 15.33 \\
\hline N. pieriae & & & 1 & 2 & & & & 3 & 1.42 & 2.00 \\
\hline N. thomsoni & & 1 & & & & & & 1 & 0.47 & 0.67 \\
\hline Nosema sp. CHW-2007a & & 2 & 1 & 2 & 1 & & 1 & 7 & 3.30 & 4.67 \\
\hline
\end{tabular}




\begin{tabular}{|c|c|c|c|c|c|c|c|c|c|c|c|c|}
\hline Sample ID & Mosquito species & A. s. & A. sp. & E. $h$. & E. a. & M. sp. PL01 & N. a. & N.c. & N. ch./p. & N. p. & N. $t$. & N. sp. \\
\hline AT.p01.C08 & O. annulipes & - & - & - & - & - & & - & 100 & 118 & & \\
\hline AT.p01.D08 & O. annulipes & - & - & - & - & - & - & - & 16 & 20 & - & - \\
\hline AT.p01.H10 & A. vexans & - & - & - & - & 38 & - & - & 584 & - & - & - \\
\hline AT.p02.A12 & O. annulipes & - & - & - & - & 15 & - & - & 15 & - & - & - \\
\hline AT.p02.C06 & \begin{tabular}{|l|} 
O. cantans \\
\end{tabular} & - & - & $\cdot$ & - & 544 & - & 16 & - & - & 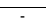 & - \\
\hline AT.p02.C07 & \begin{tabular}{|l} 
O. cantans \\
\end{tabular} & - & - & 31 & - & 6766 & - & - & - & - & - & - \\
\hline AT.p02.D06 & \begin{tabular}{|l} 
O. cantans \\
\end{tabular} & - & - & - & 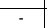 & & 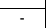 & - & 25 & - & - & 21 \\
\hline AT.p02.D12 & \begin{tabular}{|l} 
O. annulipes \\
\end{tabular} & - & - & - & 30 & 19 & 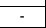 & - & & - & - & \\
\hline AT.p02.E06 & \begin{tabular}{|l} 
O. annulipes \\
\end{tabular} & - & 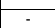 & - & - & 1283 & - & - & 3080 & - & - & 73 \\
\hline AT.p02.F05 & O. cantans & - & 14 & $=$ & - & 426 & - & - & - & - & - & 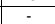 \\
\hline AT.p02.F09 & O. cantans & - & 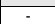 & - & - & & - & 19 & 21 & - & - & - \\
\hline AT.p02.H09 & \begin{tabular}{|l|} 
C. richiardii \\
\end{tabular} & - & - & - & - & 22 & & 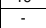 & 19 & - & - & 22 \\
\hline AT.p03.B06 & O. annulipes & - & - & - & - & 1647 & 118 & - & - & - & - & 15 \\
\hline AT.p03.C03 & O. punctor & - & - & - & - & 18 & 56 & 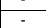 & - & - & - & \\
\hline 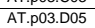 & O. sticticus & - & - & - & - & 22 & . & - & - & - & & 16 \\
\hline AT.p03.D07 & A. vexans & - & - & - & - & 8000 & - & - & - & - & 2948 & 19 \\
\hline AT.p03.D08 & A. cinereus & - & - & - & - & 3924 & 141 & - & - & - & - & \\
\hline AT.p03.E05 & \begin{tabular}{|l} 
O. cantans \\
\end{tabular} & - & - & 17 & - & 503 & - & - & - & - & - & - \\
\hline AT.p03.E07 & O. sticticus & 24 & 21 & 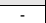 & - & - & - & - & - & - & - & - \\
\hline AT.p03.F04 & C. richiardii & & & & - & 372 & - & - & & 59 & - & \\
\hline
\end{tabular}

